\title{
Equitable Voting Rules
}

\author{
Laurent Bartholdi* Wade Hann-Caruthers ${ }^{\dagger}$ Maya Josyula \\ Omer Tamuz, and Leeat Yariv $\mathbb{q l}^{\S}$
}

February 5, 2019

\begin{abstract}
May's Theorem (1952), a celebrated result in social choice, provides the foundation for majority rule. May's crucial assumption of symmetry, often thought of as a procedural equity requirement, is violated by many choice procedures that grant voters identical roles. We show that a modification of May's symmetry assumption allows for a far richer set of rules that still treat voters equally, but have minimal winning coalitions comprising a vanishing fraction of the population. We conclude that procedural fairness can coexist with the empowerment of a small minority of individuals. Methodologically, we introduce techniques from group theory and illustrate their usefulness for the analysis of social choice questions.
\end{abstract}

*Institute of Advanced Studies, Lyon, e-mail: laurent.bartholdi@gmail.com

${ }^{\dagger}$ California Institute of Technology, e-mail: whanncar@gmail.com

${ }^{\ddagger}$ California Institute of Technology, e-mail: mjosyula@caltech.edu

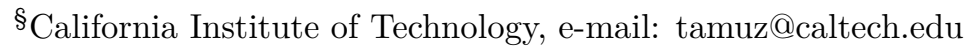

๑Princeton University, e-mail: lyariv@princeton.edu

"We thank Wolfgang Pesendorfer for useful comments. Tamuz gratefully acknowledges financial support from the Simons Foundation, through grant 419427. Yariv gratefully acknowledges financial support from the NSF, through grant SES-1629613. 


\section{Introduction}

Literally translated to "power of the people", democracy is commonly associated with two fundamental tenets: equity among individuals and responsiveness to their choices. May's celebrated theorem provides foundation for voting systems satisfying these two restrictions (May, 1952). Focusing on two-candidate elections, May illustrated that majority rule is unique among voting rules that treat candidates identically and guarantee equity and responsiveness.

Extensions of May's original results are bountiful. ${ }^{1}$ However, what we view as a procedural equity restriction in his original treatment - often termed anonymity or symmetry - has remained largely unquestioned. ${ }^{2}$ This restriction requires that no two individuals can affect the collective outcome by swapping their votes. Motivated by various real-world voting systems, this paper focuses on a particular weakening of this restriction. While still capturing the idea that no voter carries a special role, our equity notion allows for a large spectrum of voting rules, some of which are used in practice, and some of which we introduce. We analyze winning coalitions of equitable voting rules and show that they can comprise a vanishing fraction of the population. Methodologically, we demonstrate how techniques from group theory can be useful for the analysis of fundamental questions in social choice.

To illustrate our motivation, consider what we term an electoral college rule, a stylized version of the classical model of representative democracy: $m$ states each have $k$ residents. Each state selects, using majority rule, one of two representatives. Then, again using majority rule, the $m$ representatives select one of two policies (see Figure 1 for the case $m=k=3$ ).

Because all states have the same population, all residents are treated equally ex-ante. For example, if voters cast their votes independently from the same distribution, each voter has the same probability of being pivotal.

However, this seemingly equitable rule does not satisfy May's original symmetry restriction.

\footnotetext{
${ }^{1}$ See, e.g., Cantillon and Rangel (2002), Fey (2004), Goodin and List (2006), and references therein.

${ }^{2}$ An exception is Packel (1980), who relaxes the symmetry restriction and adds two additional restrictions to generate a different characterization of majority rule than May's.
} 


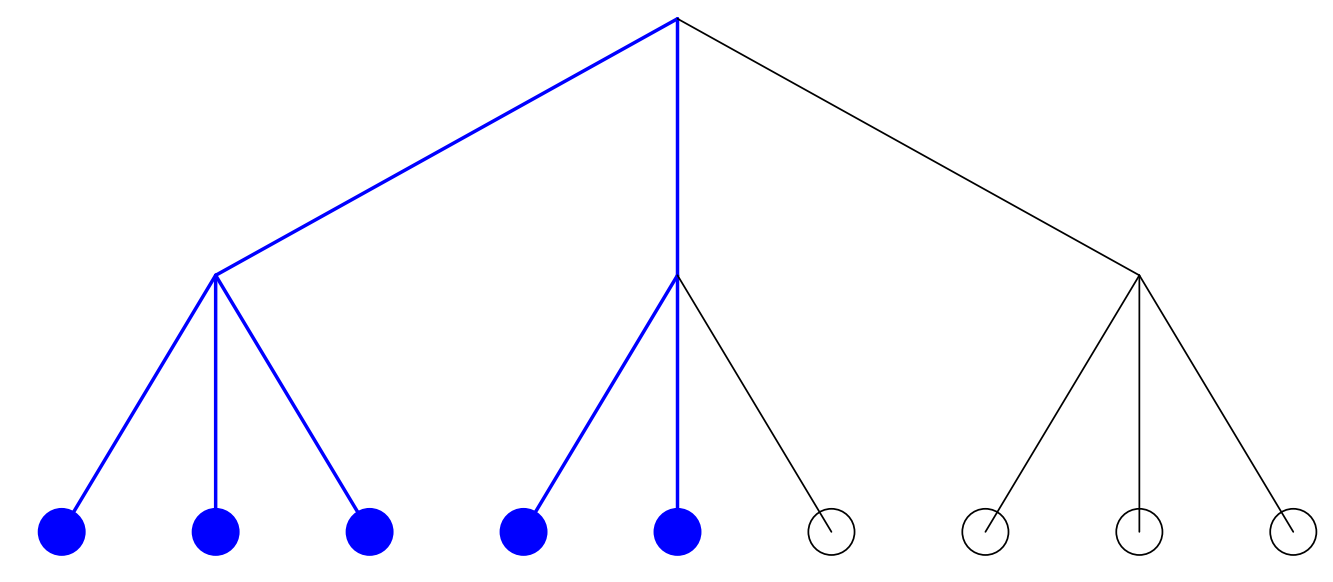

Figure 1: An electoral college voting rule. Voters are grouped into three states: $\{1,2,3\}$, $\{4,5,6\}$ and $\{7,8,9\}$. Each state elects a representative by majority rule, and the election is decided by majority rule of the representatives.

Individuals could swap their votes and change the outcome. In Figure 1, for example, suppose voters $\{1,2,3,4,5\}$ vote for representatives supporting policy A, while voters $\{6,7,8,9\}$ vote for representatives supporting policy B. With the original votes, policy A would win; but swapping voters 5 and 9 would cause policy B to win.

Even though electoral college rules do not satisfy May's symmetry assumption, their fundamental characteristics "appear" equitable. What makes a voting rule equitable? We suggest the following definition. In an equitable voting rule, every voter has the same role; that is, for any two voters $v$ and $w$, there is some permutation of the full set of voters that sends $v$ to $w$, such that applying this permutation to any voting profile leaves the election result unchanged. An implication of our equity notion is that, as in the electoral college rule, if voters cast their votes independently from the same distribution, each voter has the same probability of being pivotal.

Under this definition, electoral college rules are indeed equitable. For instance, in the case depicted in Figure 1, voters 1 and 2 clearly play the same role, since the permutation that swaps them leaves any election result unchanged. But 1 and 4 also play the same role: the 


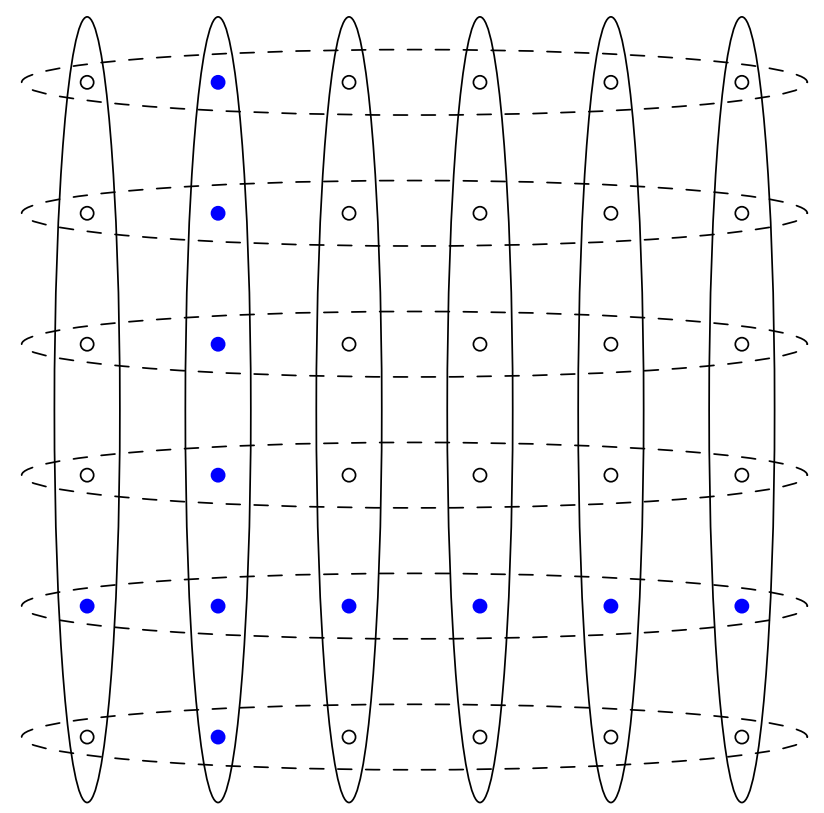

Figure 2: Cross-committee consensus voting rule. The union of a row and a column is a winning coalition.

permutation that swaps the first state with the second state also leaves outcomes unchanged.

There is a large variety of equitable rules that are not electoral college rules. An example is what we call Cross Committee Consensus ( $C C C$ ) rules. In these, each voter is assigned to two committees: a "row committee" and a "column committee" (see Figure 2). If any row committee and any column committee both exhibit consensus, then their choice is adopted. Otherwise, majority rule is followed. For instance, suppose a university is divided into equally-sized departments, and each faculty member sits on one university-wide committee. CCC corresponds to a policy being accepted if there is a strong unanimous lobby from a department and from a university-wide committee, with majority rule governing decisions otherwise. This rule is equitable since each voter is a member of precisely one committee of each type, and all row (column) committees are interchangeable.

We provide a number of further examples of equitable voting rules, showing the richness of this class and its versatility in allowing different segments of society-states, university 
departments, etc.- to express their preferences.

In order to characterize more generally the class of equitable voting rules, we focus on their winning coalitions, the sets of voters that decide the election when in agreement (Reiker, 1962). In majority rule, all winning coalitions include at least half of the population. We analyze how small winning coalitions can be in equitable voting rules.

When the number of voters $n$ is a perfect square, and when committee sizes are taken to be $\sqrt{n}$, the CCC rule has a winning coalition of size $2 \sqrt{n}-1$.

Our main result is that, for any $n$, there always exist simple equitable voting rules that have winning coalitions of size $\approx 2 \sqrt{n}$. Conversely, we show that no equitable voting rule can have winning coalitions of size less than $\sqrt{n}$. Methodologically, the proof utilizes techniques from group theory and suggests the potential usefulness of such tools for the analysis of collective choice.

May's Theorem ties equity considerations with a requirement that a majority of the population support any collectively chosen alternative. Our results suggest that procedural fairness can, in fact, coexist with the empowerment of a small minority of the population.

Interestingly, rules that give decisive power to minorities of size $\sqrt{n}$ appear in other contexts of collective choice and have been proposed for apportioning representation in the United Nations Parliamentary Assembly, and for voting in the Council of the European Union, see Życzkowski and Słomczyński (2014). ${ }^{3}$ Our results provide a general axiomatic foundation for voting rules that achieve equity among voters.

Another of our results pertains to a generalization of electoral college rules. In generalized electoral college $(G E C)$ voting rules, voters are hierarchically divided into sets (for example, states) that are, in turn, divided into subsets (for example, counties), and so on. For each set, the outcome is given by majority rule over the decisions of the subsets. ${ }^{4}$

\footnotetext{
${ }^{3}$ These proposed rules relied on the Penrose Method (Penrose, 1946), which suggests the vote weight of any representative should be the square root of the size of the population she represents, when majority rule governs decisions. Penrose argued that this rule assures equal voting powers among individuals.

${ }^{4}$ These rules have been studied under the name recursive majority in the probability literature (see, e.g., Mossel and O'Donnell, 1998).
} 
While non-equitable GEC rules can have very small winning coalitions, we show that equitable GEC rules for $n$ voters cannot have winning coalitions of size $n^{\log _{3} 2}$ or smaller. ${ }^{5}$

Certainly, beyond equity, another important aspect of voting rules is their susceptibility to manipulation. For instance, with information on voters' preferences, electoral college rules are sensitive to gerrymandering (McGann et al., 2016). We view the question of manipulability as distinct from that of equity. Our analysis sheds light on the class of equitable voting rules that can then be compared in terms of vulnerability to various manipulations.

Last, we explore a stronger notion of equity. We consider $k$-equitable voting rules in which every coalition of $k$ voters plays the same role. For $k>1$, this notion implies equity, but is not equivalent to it. The analysis of $k$-equitable rules is delicate, due to group- and number-theoretical phenomena. We show that there do exist, for arbitrarily large population sizes $n$, voting rules that are 2- and 3-equitable, and have winning coalitions as small as $\sqrt{n}$. However, for "most" sufficiently large values of $n$, and for any $k \geq 2$, the only $k$-equitable, neutral, and responsive voting rule is majority. Thus, while equity across individuals allows for a broad spectrum of voting rules, equity among arbitrary fixed-size coalitions places the restrictions May had suggested, as long as the electorate is sufficiently large. While $k$-equity is arguably a strong restriction, it is still far weaker than May's original symmetry requirement. In that respect, our results here provide a strengthening of May's conclusions.

\section{The Model}

\subsection{Voting rules}

Let $V$ be a finite set of voters. We denote $V=\{1, \ldots, n\}$ so that $n$ is the number of voters. Each voter has preferences over alternatives in the set $Y=\{-1,1\}$. We identify the possible

\footnotetext{
${ }^{5}$ For an example of a non-equitable GEC with a small winning coalitions, consider voters $\{1, \ldots, 1000\}$ and assume three states divide the population into three sets of voters: $\{\{1\},\{2\},\{3, \ldots, 1000\}$. Then $\{1,2\}$ is a winning coalition. The value $\log _{3} 2 \approx 0.63$ is the Hausdorff dimension of the Cantor set. As it turns out, there is a connection between equitable GEC's that achieve minimal winning coalitions and the Cantor set.
} 
preferences over $Y$ with elements of $X=\{-1,0,1\}$, where -1 represents a strict preference for -1 over 1,1 represents a strict preference for 1 over -1 , and 0 represents indifference between -1 and 1 . We denote by $\Phi=X^{V}$ the set of voting profiles; that is, $\Phi$ is the set of all functions from the set of voters $V$ to the set of possible preferences $X$. A voting rule is a function $f: \Phi \rightarrow X$.

An important example is the majority voting rule $\mathrm{m}: \Phi \rightarrow X$, which is given by

$$
\mathrm{m}(\phi)= \begin{cases}1 & \text { if }\left|\phi^{-1}(1)\right|>\left|\phi^{-1}(-1)\right| \\ -1 & \text { if }\left|\phi^{-1}(1)\right|<\left|\phi^{-1}(-1)\right| \\ 0 & \text { otherwise. }\end{cases}
$$

A vote of 0 can be interpreted as abstention or indifference.

\subsection{May's Theorem}

We now define several properties of voting rules. Following May (1952), we say that a voting rule $f$ is neutral if $f(-\phi)=-f(\phi)$. Neutrality implies that both alternatives -1 and 1 are treated symmetrically: if each individual flips her vote, the final outcome is also flipped.

Again following May (1952), we say that a voting rule $f$ is positively responsive if increased support for one alternative makes it more likely to be selected. Formally, $f$ is positively responsive if $f(\phi)=1$ whenever there exists a voting profile $\phi^{\prime}$ satisfying the following:

1. $f\left(\phi^{\prime}\right)=0$ or 1 .

2. $\phi(v) \geq \phi^{\prime}(v)$ for all $v \in V$.

3. $\phi\left(v_{0}\right)>\phi^{\prime}\left(v_{0}\right)$ for some $v_{0} \in V$.

Thus, $f(\phi) \geq f\left(\phi^{\prime}\right)$ if $\phi \geq \phi^{\prime}$ coordinate-wise, and if $f(\phi)=0$ then any change of $\phi$ breaks the tie.

We now turn to our notion of equity. Denote by $S_{n}$ the set of permutations of the $n$ voters. To any permutation of the voters we can associate a permutation of the set of voting 
profiles $\Phi$ : given a permutation $\sigma \in S_{n}$, the associated permutation on the voting profiles maps $\phi$ to $\phi^{\sigma}$, which is given by $\phi^{\sigma}(v)=\phi(\sigma v)$. The automorphism group of the voting rule $f$ is given by

$$
\operatorname{Aut}_{f}=\left\{\sigma \in S_{n} \mid \forall \phi \in \Phi, f\left(\phi^{\sigma}\right)=f(\phi)\right\}
$$

That is, Aut $_{f}$ is the set of permutations of the voters that leave election results unchanged, for every voting profile.

We can interpret a permutation $\sigma$ as a scheme in which each voter $v$, instead of casting her own vote, gets to decide how some other voter $w=\sigma(v)$ will vote. A permutation $\sigma$ is in Aut $_{f}$ if applying this scheme never changes the outcome: when each $w=\sigma(v)$ votes as $v$ would have, the result is the same as when each player $v$ votes for herself.

Another interpretation of Aut $f$ is through pivotality, or through the Shapley-Shubik and Banzhaf indices of players in simple games, see Dubey and Shapley (1979). Consider a setting in which all voters choose their votes identically and independently at random. Given such a distribution, we can consider the probability $\eta_{v}$ that a voter $v$ is pivotal. ${ }^{6}$ It is easy to see that if there is some $\sigma \in$ Aut $_{f}$ that maps $v$ to $w$, then $\eta_{v}=\eta_{w}$, implying that $v$ and $w$ have the same Banzhaf index. In fact, when there exists $\sigma \in$ Aut $_{f}$ that maps $v$ to $w$, any statistic associated with a voter that treats other voters identically - the probability the outcome coincides with voter $v$ 's vote, the probability that voter $v$ and another voter are pivotal, etc.-would be the same for voters $v$ and $w$.

May (1952)'s notion of equity, often termed symmetry or anonymity, requires that swapping the votes of any two voters will not affect the collective outcome. It can be succinctly stated as Aut $_{f}=S_{n}$.

May's Theorem. Majority rule is the unique symmetric, neutral, and positively responsive voting rule.

Perhaps surprisingly, the requirement of symmetry is far too strong for May's conclusions.

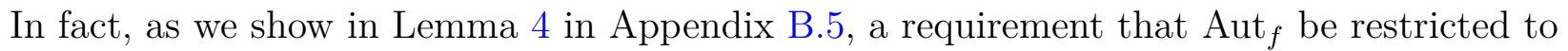

\footnotetext{
${ }^{6} \mathrm{~A}$ voter $v$ is pivotal at a particular voting profile if a change in her vote can affect the outcome under $f$.
} 
even permutations would suffice for his results. ${ }^{7}$ The set of even permutations contains half the set of all permutations.

\subsection{Equitable Voting Rules}

As we have already seen, the requirement that Aut $_{f}$ coincides with all permutations, or all even permutations, precludes many examples of voting rules that "appear" equitable. What makes a voting rule appear equitable? Our view is that, in an equitable voting rule, all voters carry the same role in the election: ex-ante, there is no reason for any voter to be envious of another. Any voter could play the role of another voter, with the proper relabeling of the electorate, without affecting outcomes. From the perspective of pivotality, if voters each have preferences that are independent and identically distributed, in an equitable voting rule, all voters should have the same probability of being pivotal. Formally, we propose the following definition.

Definition 1. A voting rule $f$ is equitable if for every $v, w \in V$ there is a $\sigma \in$ Aut $_{f}$ such that $\sigma(v)=w$.

In words, a voting rule is equitable if any two voters play the same role. That is, for any two voters $v$ and $w$, there is some permutation of the population that relabels $v$ as $w$ such that regardless of voters' preferences, the outcome is unchanged relative to the original voter labeling. This is inherently an ex-ante notion of equity. Before knowing anyone's preferences, all voters carry the same influence.

To better understand the meaning of a voting rule being equitable, consider nine voters numbered $\{1, \ldots, 9\}$, who use the simple electoral college rule depicted in Figure 1. Voters are effectively divided into three states $\{1,2,3\},\{4,5,6\}$, and $\{7,8,9\}$. Now, imagine that one learns voters' names, as well as their state, but not their associated numbers. For example,

\footnotetext{
${ }^{7} \mathrm{~A}$ permutation $\sigma$ is even if the number of pairs $(v, w)$ such that $v<w$ and $\sigma(v)>\sigma(w)$ is even. Put another way, define a transposition to be a permutation that only switches two elements, leaving the rest unchanged. A permutation is even if it is the composition of an even number of transpositions.
} 
suppose that Alex, Andrea, and Avery are in one state, that Bailey, Blair, and Brooke are in another, and that Carter, Casey, and Channing are in yet another. The rule being equitable is tantamount to it being impossible to deduce, from the outcomes of every voting profile, the number associated with any given name. Indeed, such information is consistent with, say, Alex taking any role in $\{1, \ldots, 9\}$.

In group-theoretic terms, $f$ is equitable if and only if the group Aut $_{f}$ is transitive. ${ }^{8}$ Insights from group theory related to the characteristics of transitive groups are therefore at the heart of our main results. Appendix A contains a short primer on the basic group theoretical results that are useful for our analysis.

\subsection{Winning coalitions}

One way to describe a voting rule is through its winning coalitions, the sets of individuals whose consensual vote determines the alternative chosen. Formally, we say that a subset $S \subseteq V$ is a winning coalition with respect to the voting rule $f$ if, for every voting profile $\phi$ and $x \in\{-1,1\}, \phi(v)=x$ for all $v \in S$ implies $f(\phi)=x$.

Under majority rule, all winning coalitions are of size $n / 2$. Our results will show that relaxed equity requirements allow for voting rules with far smaller winning coalitions.

Note that no two winning coalitions of $f$ can be disjoint. Indeed, suppose that $M, M^{\prime} \subseteq V$ are two disjoint winning coalitions. We can then have a profile under which members of $M$ vote unanimously for -1 and members of $M^{\prime}$ vote for 1 . In such cases, $f$ would not be well-defined. The following lemma illustrates a version of the reverse.

Lemma 1. Let $\mathcal{W}$ be a collection of subsets of $V$ such that every pair of subsets in $\mathcal{W}$ has nonempty intersection. Then there is a neutral, positively responsive voting rule for $V$ for which every set in $\mathcal{W}$ is a winning coalition.

Intuitively, the construction underlying Lemma 1 is as follows. First, for any vote profile in which a winning coalition votes for 1 (or -1 ) in consensus, we specify the voting rule

\footnotetext{
${ }^{8}$ It turns out our equity restriction is effectively the definition of transitivity. The notion of transitive groups is not directly related to transitivity of relations often considered in Economics.
} 
to also take the value of 1 (or -1 ). For any profile in which no winning coalition votes in consensus, we define the voting rule to follow majority rule. By definition, the winning coalitions of this voting rule contain the subsets we started out with. As we show, it is also neutral and positively responsive.

This lemma will allow us to discuss neutral and positive-responsive equitable voting rules through the restrictions they impose on winning coalitions.

\section{Winning Coalitions for Equitable Voting Rules}

In this section, we provide bounds on the size of winning coalitions in general equitable voting rules. We then restrict attention to the special class of equitable voting rules that generalize electoral college rules and characterize the size of winning coalitions for those.

\section{$3.1 \quad$ Winning Coalitions of Order $\sqrt{\mathbf{n}}$}

We first illustrate that for any population size, there always exist equitable voting rules that have winning coalitions that are far smaller than $n / 2$.

Theorem 1. For every $n$ there exists a neutral, positively responsive equitable voting rule with winning coalitions of size at most $2 \sqrt{n}+2$.

An important implication of the Theorem is that, under an equitable voting rule, winning coalitions can account for a vanishing fraction of the population. In that respect, procedural equity can go hand in hand with the empowerment of a small minority of the population.

The cross committee consensus rule described in the Introduction is an example of an equitable voting rule in which winning coalitions are $O(\sqrt{n})$. However, there is an algebraic subtlety - the construction of that rule relies on $n$ being an integer squared. Certainly, an analogous construction can be made for any $n$ that can be described as $n=k \cdot m$ for some integers $k$ and $m$ by considering some committees to be of size $k$ and others to be of size

$m$. Such constructions, however, would not necessarily generate voting rules with winning coalitions of size close to $\sqrt{n}$. 


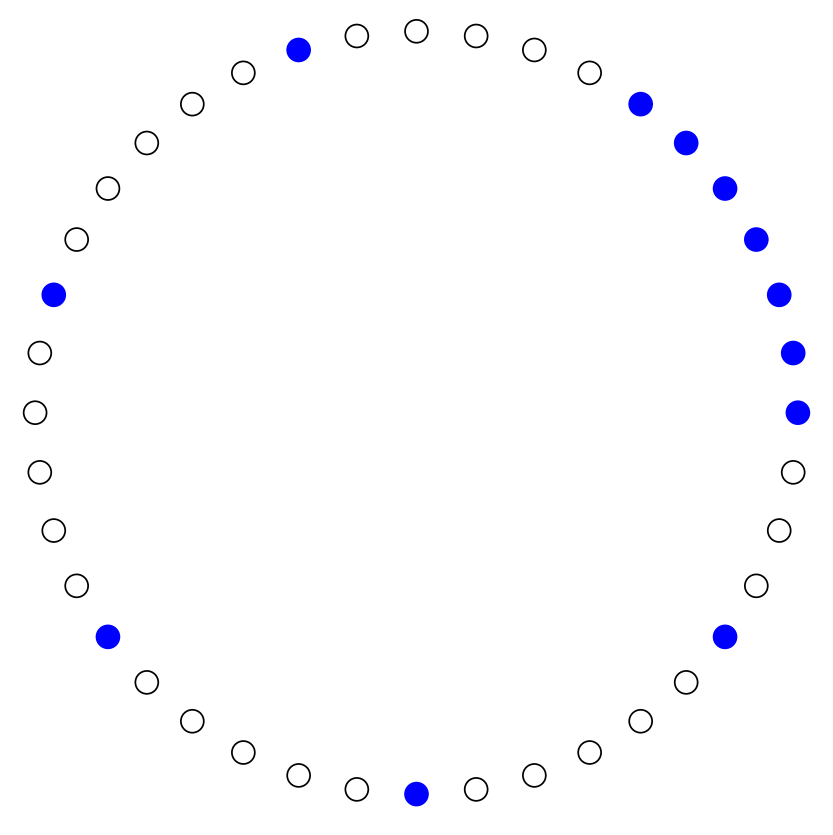

Figure 3: A cyclic voting rule.

Nonetheless, there is a simple class of rules that justifies the result in Theorem 1. A voting rule $f: \Phi \rightarrow X$ is a cyclic voting rule if there is a $\sigma \in$ Aut $_{f}$ such that for any $a, b \in V$ there is some $i \in \mathbb{N}$ such that $\sigma^{i}(a)=b$.

Intuitively, a voting rule is cyclic if the voters can be arranged on a circle in such a way that if all voters shift to the right the same number of spaces and vote the same way, the outcome of the vote is the same. An example of a cyclic voting rule is illustrated in Figure 3. Formally, the rule in this example is defined as follows:

- For any $n$, denote $k=\lceil\sqrt{n}$. We mark in blue the subset of the voters $\{1, \ldots, n\}$ given by $\{1,2, \ldots, k\}$, as well as those that are equal to 0 modulus $k$.

- If all voters marked blue vote for $x \in\{-1,1\}$, then $x$ is the outcome.

- Similarly, if all voters who are exactly $i$ spaces to the right of a voter marked blue vote for $x \in\{-1,1\}$, then $x$ is the outcome.

- If neither of the above conditions obtains, then the outcome is decided by majority. 
If proximity on the circle signifies similarity on some relevant dimension - field of interest in a university, specialty in a firm, etc.- this rule requires winning coalitions to be composed of a sufficiently large lobby of similar individuals together with a sufficiently large lobby of diverse individuals. In that respect, this rule bears a resemblance to the cross committee consensus rule, which also requires two coalition types to agree in order to guarantee an outcome.

Cyclic voting rules are equitable by construction. Indeed, if any individual $i$ desires the role of individual $j$, a permutation defined by a rotation of individuals that leaves individual $i$ in $j$ 's position would certainly render outcomes unchanged. Furthermore, this example illustrates that, for every electorate size $n$, there exist cyclic voting rules with winning coalitions that entail $O(\sqrt{n})$ members. $^{9}$

We now provide a lower bound on the size of minimal coalitions in equitable voting rules. While this bound is far smaller than $n / 2$, it is still substantial.

Theorem 2. Every winning coalition of an equitable voting rule has size at least $\sqrt{n}$.

The lower bound of $\sqrt{n}$ on a winning coalition's size is tight: in the proof of Theorem 6 in Appendix C, we construct, for arbitrarily large $n$, an equitable voting rule that has winning coalitions of size exactly $\sqrt{n}$, rounded up to the nearest integer.

The proof of Theorem 2 relies on group-theoretic results described in Appendix A. To gain some intuition for the bound, suppose, as in the example above, that voters are located on a circle and that $\mathrm{Aut}_{f}$ corresponds to all rotations. These are permutations that relabel voter $v$ as voter $v+x$ (modulo $n$ ) for some $x=0,1, \ldots, n-1$. We know that two winning coalitions cannot be disjoint. Take, then, any winning coalition $S$ and denote by $S+x$ the winning coalition that is derived by adding $x$ (again, modulo $n$ ) to the label of each member. It follows that $S$ and $S+x$ must have a non-empty intersection. Therefore, if we look at all the differences between two elements of $S$ (i.e., elements of the form $a-b$, where $a, b \in S$ ), they encompass all permutations in $\mathrm{Aut}_{f}$. In particular, the cardinality of these differences is

\footnotetext{
${ }^{9}$ When the number of voters is prime, every equitable voting rule is cyclic. Hence, in some sense, cyclic voting rules are an important class among equitable rules, as they are guaranteed to exist for all $n$.
} 
$n$. On the other hand, the number of such differences is certainly bounded by the number of ordered pairs of members in $S$, which is $|S|^{2}$. It follows that $|S|^{2} \geq n$, generating our bound.

\subsection{Generalized Electoral College Rules}

Our discussion in the introduction suggested that voting rules mimicking the U.S. electoral college are equitable, if not symmetric à la May (1952). Formally, a voting rule $f: \Phi \rightarrow X$ is a generalized electoral college $(G E C)$ if the following holds.

- If $V=\{v\}$ is a singleton, then $f(\phi)=\phi(v)$.

- If $V$ is not a singleton, there exists a partition $\left\{V_{1}, \ldots, V_{d}\right\}$ of $V$ into $d$ sets such that

$$
f(\phi)=\mathrm{m}\left(f_{1}\left(\left.\phi\right|_{V_{1}}\right), f_{2}\left(\left.\phi\right|_{V_{2}}\right), \ldots, f_{d}\left(\left.\phi\right|_{V_{d}}\right)\right)
$$

where each $f_{i}: X^{V_{i}} \rightarrow X$ is some generalized electoral college rule, $\left.\phi\right|_{V_{i}}$ is $\phi$ restricted to $V_{i}$, and $\mathrm{m}$ is the majority rule.

Any GEC rule is associated with a rooted tree that captures voters' hierarchical structure (as in Figure 4 for the case of $d=3$ ). A GEC voting rule is equitable if, in the induced tree, the vertices in each level have the same degree. ${ }^{10}$

The following result characterizes the size of winning coalitions in GEC voting rules.

Theorem 3. If $f$ is an equitable generalized electoral college rule for $n$ voters, then a winning coalition must have size of at least $n^{\log _{3} 2}$. Conversely, for arbitrarily large $n$, there exist equitable generalized electoral college voting rules with winning coalitions of size $n^{\log _{3} 2}$.

There is an intriguing connection between this characterization and the so-called Hausdorff dimension of the Cantor set ${ }^{11}$, which is $\log _{3} 2 \approx 0.63$. The connection arises from the fact

\footnotetext{
${ }^{10}$ Intuitively, the permutations required to shift one voter's role into another require the shift of that voter's entire "state" into the target role's "state", which can be done only when their numbers coincide.

${ }^{11}$ The Cantor set can be constructed by starting from, say, the unit interval and iteratively deleting the open middle third of any sub-interval remaining. That is, in the first iteration we are left with $[0,1 / 3] \cup[2 / 3,1]$, in the second iteration we are left with $[0,1 / 9] \cup[2 / 9,1 / 3] \cup[2 / 3,7 / 9] \cup[8 / 9,1]$, etc. The fractal or Hausdorff dimension is a measure of "roughness" of a set. See Peitgen et al. (1993) and references therein.
} 


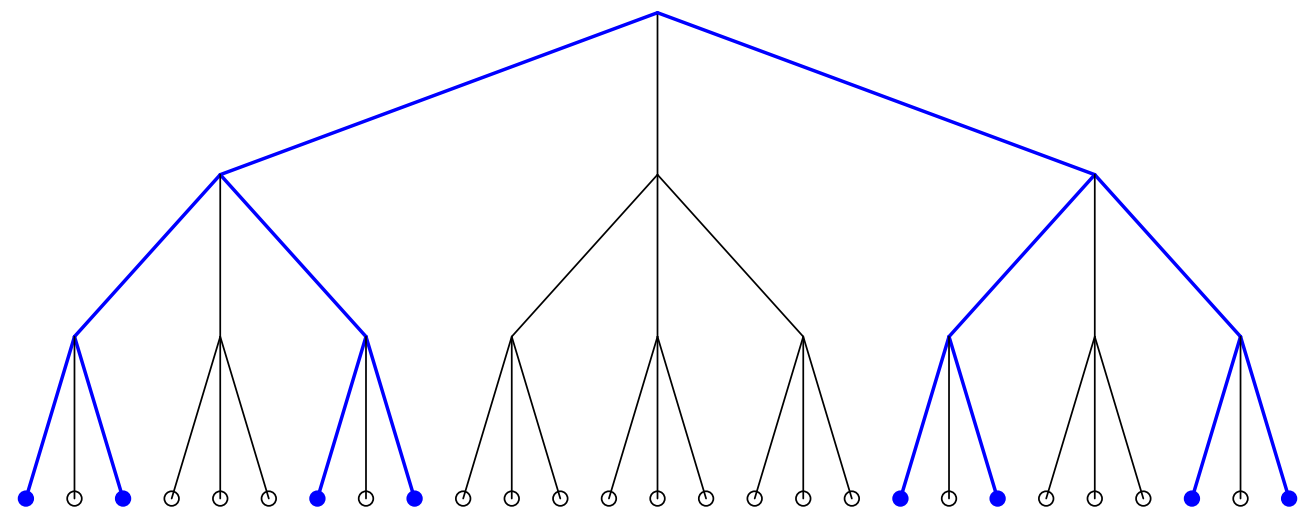

Figure 4: Generalized electoral college voting rule. The leafs of the tree (at the bottom) represent the voters. At each intermediate node the results of the three nodes below are aggregated by majority.

that GEC rules with the smallest winning coalitions are those in which, at each level, the subdivision is into three groups. In such rules, to construct a winning coalition, two of the three top "representatives" need to agree. Then, two of the voters of these representatives need to agree, and so on recursively. This precisely mimics the classical construction of the Cantor set.

\section{4 k-Equitable Voting Rules}

So far, we have been focusing on voting rules that are robust to any individual switching roles. Naturally, one could extend the notion and contemplate rules that are robust to larger coalitions of voters changing their roles in the population. This section analyzes such rules for arbitrary size $k$ of coalitions. With such harsher restrictions on collective-choice procedures, results similar to May's reemerge, though with important caveats.

Definition 2. A voting rule is $k$-equitable for $k \geq 1$ if for every pair of ordered $k$-tuples $\left(v_{1}, \ldots, v_{k}\right)$ and $\left(w_{1}, \ldots, w_{k}\right)$ (with $v_{i} \neq v_{j}$ and $w_{i} \neq w_{j}$ for all $\left.i \neq j\right)$, there is a permutation $\sigma \in$ Aut $_{f}$ such that $\sigma\left(v_{i}\right)=w_{i}$ for $i=1, \ldots, k$. 
Intuitively, $k$-equitable voting rules are ones in which every group of $k$ voters has the same role in the election. This restriction is certainly harsher than that imposed for equitable rules. Indeed, consider the example above corresponding to Figure 1. 2-equity implies that, say, the pair (Alex, Andrea) could potentially be associated with any pair $(i, j)$. But this is clearly not true here, since Alex and Andrea are in the same state, and thus it is impossible that, e.g., they are associated with the numbers 1 and 4 .

In group-theoretic terms, $f$ is $k$-equitable if and only if the group Aut $_{f}$ is $k$-transitive. The group of permutations of a finite set of size $n$ is certainly $k$-transitive, as long as $n \geq k$. The group of even permutations of a set of size $n$ is also easily seen to be $k$-transitive. ${ }^{12}$

At the heart of our analysis of $k$-equitable voting rules is the observation that when $\mathrm{Aut}_{f}$ is the group of even permutations, every winning coalition must be of size at least $n / 2$ (Lemma 4).

Certainly, majority rule is 2-equitable. As can be easily verified, none of the voting-rule examples mentioned so far, other than majority, is 2-equitable. As it turns out, for most population sizes, large winning coalitions, of size of at least $n / 2$, are endemic to 2-equitable voting rules.

We say that almost every natural number satisfies a property $P$ if the subset $N_{P} \subseteq \mathbb{N}$ of the natural numbers that have property $P$ satisfies

$$
\lim _{n \rightarrow \infty} \frac{\left|N_{P} \cap\{1, \ldots, n\}\right|}{n}=1 .
$$

Theorem 4. For almost every natural number $n$, every 2 -equitable voting rule for $n$ voters has no winning coalitions of size less than $n / 2$. In particular, for almost all $n$, the only 2-equitable, neutral, positively responsive voting rule is majority.

The proof of Theorem 4 relies on group-theoretical results (Cameron et al., 1982). As it turns out, there is a vanishing share of integers for which there exist 2-transitive groups that

\footnotetext{
${ }^{12}$ Identifying the set of voters with the numbers $1, \ldots, n$, recall that a permutation $\sigma$ is even if the number of pairs $(i, j)$ such that $i<j$ and $\sigma(i)>\sigma(j)$ is even.
} 
are neither the set of all permutations nor the set of even permutations. Since those latter groups yield winning coalitions of size at least $n / 2$, the result follows.

While Theorem 4 suggests that for most population sizes, 2-equitable rules imply large winning coalitions, we construct 2-equitable rules with small winning coalitions. A general construction of such rules is rather technically involved and uses finite vector spaces. To glean some intuition, we explain an analogous construction using standard vector spaces and assuming a continuum of voters.

Suppose voters are identified with the set of one-dimensional subspaces of $\mathbb{R}^{3}$ : i.e., each voter is identified with a line that passes through the origin. Now suppose winning coalitions are the two-dimensional subspaces: if all voters on a plane agree, that is the election outcome, otherwise the election is undecided. ${ }^{13}$ Clearly, the winning coalitions are much smaller than the electorate (or indeed of "half of the voters") in the sense that they have a smaller dimension.

Invertible linear transformations of $\mathbb{R}^{3}$ permute the one-dimensional subspaces, and the two-dimensional subspaces, and so constitute automorphisms of this voting rule. Equity follows since for any two non-zero vectors $v$ and $u$, we can find some invertible linear transformation that maps $v$ to $u$. Moreover, the voting rule is also 2-equitable - given a pair of distinct voters $\left(v_{1}, v_{2}\right)$, and given another such pair $\left(u_{1}, u_{2}\right)$, we can find some invertible linear transformation that maps the former to the latter. Thus, every pair of voters plays the same role.

Our construction of 2-equitable voting rules for finite sets of voters is identical, using finite vector spaces instead of $\mathbb{R}^{3}$ (see Theorem 6). Figure 5 shows a 2-equitable voting rule constructed in this way, for 7 voters. In the figure, every three co-linear nodes form a winning coalition, as well as the three nodes on the circle. ${ }^{14}$ In this construction, the size of the winning coalition is exactly $\sqrt{n}$ (rounded up to the nearest integer), which matches the lower bound of $\sqrt{n}$ in Theorem 2 .

\footnotetext{
${ }^{13}$ This rule is well defined since every pair of two-dimensional subspaces intersects, and so no two winning coalitions are disjoint.

${ }^{14}$ Figure 5 depicts what is commonly referred to as a Fano plane in finite geometry. It is the finite projective plane of order 2.
} 


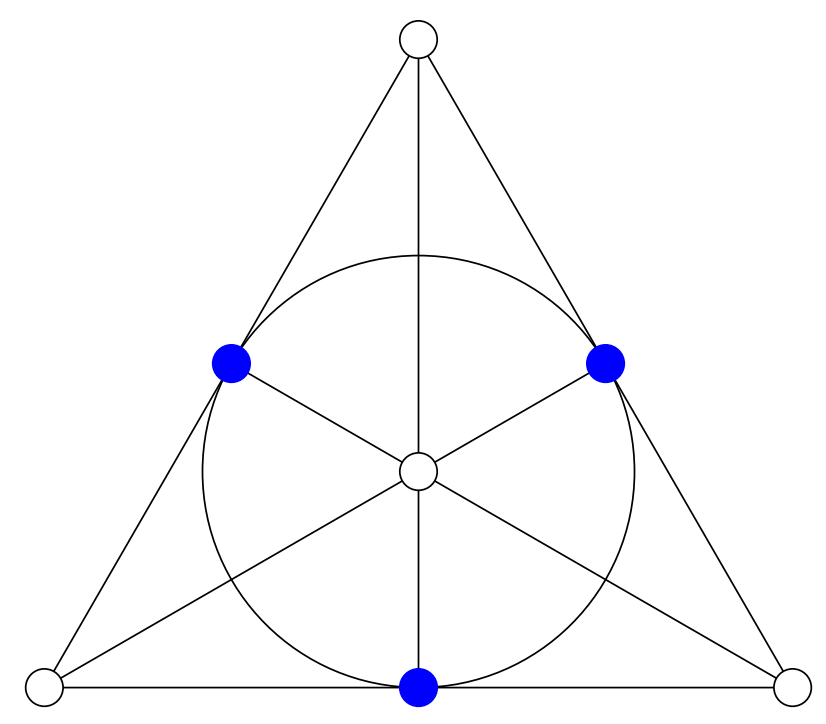

Figure 5: Every three co-linear points form a winning coalition, as well as the three points on the circle (marked in blue). This voting rule is 2-equitable.

Certainly, one can replicate this construction for arbitrarily large $n$. For the interested reader, precise constructions of 2- as well as 3-equitable rules for arbitrarily large populations appear in Appendix C. However, because of number-theoretical issues, this construction applies only for particular electorate sizes. ${ }^{15}$ These restrictions are then consistent with the message of Theorem 4, that for most possible sizes $n$ of the electorate, every winning coalition of a 2-equitable rule must be of size at least $n / 2$.

Since 3-equitable rules are, in particular, 2-equitable, the result of Theorem 4 naturally follows for them as well, and for almost all population sizes, though not all - see Appendix C for exceptions - winning coalitions contain at least $n / 2$ individuals.

When considering harsher equity restrictions, results are much starker and conclusions hold for all population sizes large enough:

Theorem 5. There exists $n^{*}$ such that for any $n \geq n^{*}$, for any $k \geq 4$, every $k$-equitable voting rule has no winning coalitions of size less than $n / 2$. In particular, the only $k$-equitable, neutral, positively responsive voting rule is majority.

\footnotetext{
${ }^{15}$ The set of voters has to be of size $q^{2 k}+q^{k}+1$, for some $k \geq 1$ and prime $q$.
} 
Theorem 5 follows from the following observations. As mentioned already, the group of all permutations of a finite set of size $n$ is certainly $k$-transitive, as long as $n \geq k$. The group of even permutations of a set of size $n$ is also easily seen to be $k$-transitive in this case. Here we rely on discoveries from the 1980's and 1990's that showed these to be the only examples of groups that are 6 -transitive and hence $k$-transitive for any $k \geq 6$. Moreover, it was discovered that there are only finitely many other groups that are 4-transitive or 5 -transitive. These results are a consequence of the successful completion of a large project, involving thousands of papers and hundreds of authors, called the Classification of Finite Simple Groups, see Dixon and Mortimer (1996).

As mentioned, we show that when Aut $_{f}$ is the group of even permutations, every winning coalition must be of size at least $n / 2$ (Lemma 4). It thus follows that, except for a finite number of possible population sizes (that are in fact all at most 24), any 4-equitable voting rule must have winning coalitions of size at least $n / 2$.

We stress that $k$-equity is a strong restriction. It requires that arbitrary coalitions of a fixed size play the same role in the election, reminiscent of group-envy-freeness notions considered in allocation problems (see, e.g., Varian, 1974). Nonetheless, for any fixed $k$, $k$-equity is a far weaker restriction than May's symmetry restriction. In that respect, Theorem 5 offers a strengthening of May's original result.

\section{Conclusions}

This paper suggests that procedural equity in collective choice can go hand in hand with the empowerment of a small set of individuals. We believe the approach taken here could potentially be useful for various other contexts. For example, symmetric games are often thought of as ones in which any permutation of players' identities does not affect individual payoffs (e.g., Dasgupta and Maskin, 1986, page 18). As is well known, such finite games have symmetric equilibria. Interestingly, in his original treatise on games, Nash took an approach to symmetry that is similar to ours, studying the automorphism group of the game. He 
showed that equity, analogously defined for games, suffices for the existence of symmetric equilibria: that is, it suffices that for every two players $v$ and $w$ there is an automorphism of the game that maps $v$ to $w$ (Nash, 1951, page 289). It would be interesting to explore further the consequences of equity so defined in more general strategic interactions.

\section{A A Primer on Finite Groups}

This section contains what is essentially a condensed first chapter of a book on finite groups (see, e.g., Rotman, 2012), and is provided for the benefit of readers who are not familiar with the topic. The terms and results covered here suffice to prove the main results of this paper.

Denote by $N=\{1, \ldots, n\}$. A permutation of $N$ is a bijection $g: N \rightarrow N$. The inverse of a permutation $g$ is denoted by $g^{-1}\left(\right.$ so $\left.g^{-1}(g(i))=i\right)$, and the composition of two permutations $g$ and $h$ is simply $g h$; i.e., if $k=g h$ then $k(i)=g(h(i))$.

A group - for our purposes - will be a non-empty set of permutations that (1) contains $g^{-1}$ whenever it contains $g$, and (2) contains $g h$ whenever it contains both $g$ and $h$.

Groups often appear as sets of permutations that preserve some invariant. In our case, $\mathrm{Aut}_{f}$ is the group of permutations of the voters that preserves every outcome of $f$. It is easy to see that $\mathrm{Aut}_{f}$ is indeed a group.

A subgroup $H$ of $G$ is simply a subset of $G$ that is also a group. Given $g \in G$, we denote

$$
g H=\{g h \in G: h \in H\}
$$

The sets $g H$ are in general not subgroups, and are called the left cosets of $H$ (the right cosets are of the form $\mathrm{Hg}$ ). It is easy to verify that all left cosets are disjoint, and that each has the same size as $H$. It follows that the size of $G$ is divisible by the size of $H$.

Given an element $i \in N$, we denote by $G_{i}$ the set of permutations that fix $i$. That is, $g \in G_{i}$ if $g(i)=i . G_{i}$ is a subgroup of $G$. It is called the stabilizer of $i$.

The $G$-orbit of $i \in N$ is the set of $j \in N$ such that $j=g(i)$ for some $g \in G$. As it turns out, if $j$ is in the orbit of $i$ then the set of $g \in G$ such that $g(i)=j$ is a coset of the stabilizer 
$G_{i}$. It follows that there is a bijection between the orbit of $i$ and the cosets of $G_{i}$. This is called the Orbit-Stabilizer Theorem.

Recall that $G$ is transitive if for all $i, j$ there is a $g \in G$ such that $g(i)=j$. This is equivalent to there existing only a single $G$-orbit, or that $j$ is in the orbit of $i$ for every $i, j$. Therefore, if $G$ is transitive, the orbit of $i$ is of size $n$, and since we can identify the orbit with the cosets of $G_{i}$, there are $n$ such cosets. Since they are all the same size as $G_{i}$, and since they form a partition of $G$, each coset of $G_{i}$ must be of size $|G| / n$. We will use this fact in the proof of Theorem 2.

\section{B Proofs}

\section{B.1 Proof of Lemma 1}

Proof of Lemma 1. Let $f$ be the voting rule defined as follows. For a voting profile $\phi$, if there is a set $W \in \mathcal{W}$ such that $\phi(w)=1$ for all $w \in W$, then $f(\phi)=1$, and similarly, if there is a set $W \in \mathcal{W}$ such that $\phi(w)=-1$ for all $w \in W$, then $f(\phi)=-1$. (Note that this is well-defined, since if there are two such sets $W$, they must agree because they intersect.) If not, then $f(\phi)$ is determined by majority.

That $f$ is neutral follows immediately from the symmetry in the definition of $f$ when some $W \in \mathcal{W}$ agrees on either 1 or -1 and the fact that majority is neutral. To see that $f$ is positively responsive, suppose that $f(\phi) \in\{0,1\}, \phi^{\prime}(x) \geq \phi(x)$ for all $x \in V$, and $\phi^{\prime}(y)>\phi(y)$ for some $y \in V$. Since $f(\phi) \neq-1$, there is no set $W \in \mathcal{W}$ such that $\phi(x)=-1$ for all $x \in W$, hence the same is true for $\phi^{\prime}$. If there is some set $W \in \mathcal{W}$ such that $\phi^{\prime}(x)=1$ for all $x \in W$, then $f\left(\phi^{\prime}\right)=1$. If not, then the same is true of $\phi$, and hence by positive responsiveness of majority, $f\left(\phi^{\prime}\right)=1$.

Finally, it is immediate from the definition of $f$ that every $W \in \mathcal{W}$ is a winning coalition. 


\section{B.2 Proof of Theorem 1}

In this proof we identify the set of voters with $\{0,1, \ldots, n-1\}$, the set of integers modulo $n$. We can perform addition and multiplication on this set, which will be taken modulo $n$, so that the result is always in this set.

Let $m=\lfloor\sqrt{n}\rfloor$, and consider the set of voters $S=\{0,1, \ldots, m\} \cup\{2 m, 3 m, \ldots, m \cdot m,(m+$ 1) $m,(m+2) m\}$. Note that since we consider the integers modulo $n,(m+2) m$ is still an element of the set of voters.

The rotations of the set of voters is the group of cyclic permutations. Formally, the group of rotations is identified with the integers modulo $n$ (coinciding with the set of voters). The application of a permutation $x \in\{0, \ldots, n-1\}$ to a voter $i$ is simply $i+x$ modulo $n$.

We will construct a voting rule $f$ that is invariant to rotations. The set $S$ described above will be a winning coalition, as will all of its rotations $S+x$. The next lemma shows that these winning coalitions are pairwise non-disjoint.

Lemma 2. $S \cap(S+x) \neq \emptyset$ for all rotations $x$.

Proof. Fix a rotation $x \in\{0, \ldots, n-1\}$.

If $x<m$, then $x=0+x$, so $x \in S \cap(S+x)$, and thus $S \cap(S+x)$ is not empty.

If $m \leq x<(m+2) m$, then write $x=k m+j$ with $0 \leq j<m, 0<k \leq m+1$. Then $m-j \in S \Longrightarrow(m-j)+x \in S+x$ and $(m-j)+x=(k+1) m \in S$, so $(m-j)+x \in S \cap(S+x)$.

If $x \geq(m+2) m$, then $(m+2) m \geq(\sqrt{n}+1)(\sqrt{n}-1)=n-1$, so $x=n-1$. In this case, $1 \in S \Longrightarrow 1+x=0 \in S+x$, so $0 \in S \cap(S+x)$.

We have thus constructed a set of voters $S$ of size at most $2 m+2 \leq 2 \sqrt{n}+2$ that intersects all of its rotations. Given this, the proof of Theorem 1 follows.

Proof of Theorem 1. Let $\mathcal{W}$ be the collection of sets of the form $S+x$ for some rotation $x$. Lemma 2 shows that these are pairwise non-disjoint, and so by Lemma 1 we have a neutral, positively responsive voting rule with winning coalitions of size at most $2 \sqrt{n}+2$. Moreover, 
this rule is equitable since each rotation is in its automorphism group, and since for each pair of voters $i, j$ there is a rotation $x=j-i$ that maps $i$ to $j$.

\section{B.3 Proof of Theorem 2}

Readers who are not familiar with the theory of finite groups are encouraged to read Section A before reading this proof.

Recall that the group of all permutations of a set of size $n$ is denoted by $S_{n}$.

The next lemma shows that if a group $G$ acts transitively on $\{1, \ldots, n\}$, then any set $S$ that intersects all of its translates (i.e., sets of the form $g S$ for $g \in G$ ) must be of size at least $\sqrt{n}$. The proof of the theorem will apply this lemma to a winning coalition $S$.

Lemma 3. Let $G \subset S_{n}$ be transitive, and suppose that $S \subseteq V$ is such that for all $g \in G$, $g S \cap S \neq \emptyset$. Then $|S| \geq \sqrt{n}$.

Proof. For any $v, w \in V$, define $\Gamma_{v, w}=\{g \in G: g(v)=w\}$. Then $\Gamma_{v, w}$ is a left coset of the stabilizer of $v$. Hence, and since the action is transitive, it follows from the orbit-stabilizer theorem that $\left|\Gamma_{v, w}\right|=\frac{|G|}{n}$. If $g S \cap S \neq \emptyset$ for all $g \in G$, then for any $g \in G$, there exists $v, w \in S$ such that $g(v)=w$, hence

$$
\bigcup_{v, w \in S} \Gamma_{v, w}=G
$$

So

$$
|G|=\left|\bigcup_{v, w} \Gamma_{v, w}\right| \leq \sum_{v, w}\left|\Gamma_{v, w}\right|=|S|^{2} \frac{|G|}{n},
$$

and we conclude that $|S| \geq \sqrt{n}$.

Our lower bound (Theorem 2) is an immediate corollary of this claim.

Proof of Theorem 2. Let $f$ be an equitable voting rule for the set of voters $V$, and suppose that $W \subseteq V$ is a winning coalition for $f$. Then for every $\sigma \in \mathrm{Aut}_{f}$, it must be the case that $\sigma(W) \cap W \neq \emptyset$ (if this failed, $f$ would not be well-defined.) Hence, it follows from Lemma 3 that $|W| \geq \sqrt{n}$. 


\section{B.4 Proof of Theorem 3}

Proof of Theorem 3. Define $C(n)$ to be the smallest size of any winning coalition in any generalized electoral college rule for $n$ voters. We want to show that $C(n) \geq n^{\log _{3} 2}$.

If $n=1$ then a winning coalition must be of size 1 , which is $\geq 1^{\log _{3} 2}$.

If $n>1$, then any generalized voting rule $f$ is of the form $f(\phi)=\mathrm{m}\left(f_{1}\left(\left.\phi\right|_{V_{1}}\right), f_{2}\left(\left.\phi\right|_{V_{2}}\right), \ldots, f_{d}\left(\left.\phi\right|_{V_{d}}\right)\right)$. Because the voting rule is equitable, any minimal winning coalition for each of the $f_{i}$ would be of the same size. A minimal winning coalition for $f$ would then need to include a strict majority of these, which is of size at least $\frac{d+1}{2}$. So

$$
C(n) \geq \min _{d \mid n} \frac{d+1}{2} \cdot C(n / d) .
$$

Assume by induction that $C(m) \geq m^{\log _{3} 2}$ for all $m<n$. Then for $d \mid n$,

$$
\frac{d+1}{2} \cdot C(n / d) \geq \frac{d+1}{2} \cdot\left(\frac{n}{d}\right)^{\log _{3} 2}=n^{\log _{3} 2} \cdot \frac{d+1}{2} \cdot d^{-\log _{3} 2} .
$$

Denote $h(d)=\frac{d+1}{2} \cdot d^{-\log _{3} 2}$, so that

$$
\frac{d+1}{2} C(n / d) \geq n^{\log _{3} 2} h(d) .
$$

Note that $h(d) \geq 1$. To see this, observe that $h(3)=1$, and

$$
h^{\prime}(d)=\frac{d^{-\log 6 / \log 3}\left(d \log \frac{3}{2}-\log 2\right)}{2 \log 3}>0
$$

for $d \geq 3$, and so $h(d) \geq 1$ for $d \geq 3$.

We have thus shown that

$$
\frac{d+1}{2} C(n / d) \geq n^{\log _{3} 2},
$$

and so by (1), $f(n) \geq n^{\log _{3} 2}$.

For the other direction, take $n$ to be a power of 3 , and let $f$ be defined recursively by choosing the partitions $V_{1}, \ldots, V_{d}$ to be of size $d=3$. A simple calculation shows that the winning coalition recursively consisting of the winning coalitions of $V_{1}$ and $V_{3}$ is of size $n^{\log _{3} 2}$. 


\section{B.5 Proof of Theorems 4 and 5}

The group of all even permutations is called the alternating group and is denoted $A_{n}$.

Lemma 4. Let $f$ be a voting rule $n$ voters. If $\operatorname{Aut}_{f}$ is either $S_{n}$ or $A_{n}$ then every winning coalition for $f$ has size at least $n / 2$.

Proof. Suppose $W \subseteq V$ is a winning coalition for $f$ with $|W|=k<n / 2$. Label the voters $V$ with labels $1, \ldots, n$ such that $W=\{1, \ldots, k\}$, and let $\pi$ be the permutation of $V$ given by $\pi(i)=n+1-i$ for $i=1, \ldots, n$. If $\lfloor n / 2\rfloor$ is odd, then let $\pi$ be the map above composed with the map that exchanges 1 and 2. Then $\pi$ is in the alternating group, and hence $\pi \in \mathrm{Aut}_{f}$. However, $\pi(W) \cap W=\emptyset$ since $k<n+1-k$, contradicting the assumption $W$ is a winning coalition.

Proof of Theorem 4. Denote by $\eta(n)$ the number of positive integers $m \leq n$ for which there is no 2-transitive group action on a set of $m$ elements except for $S_{m}$ and $A_{m}$. It follows from the main theorem in Cameron et al. (1982) that $n-\eta(n)$ is at most $3 n / \log (n)$ for all $n$ large enough. Since

$$
\lim _{n \rightarrow \infty} \frac{3 n / \log (n)}{n}=0,
$$

it follows that

$$
\lim _{n \rightarrow \infty} \frac{\eta(n)}{n}=\lim _{n \rightarrow \infty} 1-\frac{n-\eta(n)}{n}=1,
$$

and so the claim follows from Lemma 4 .

Proof of Theorem 5. The only 4- or 5-transitive finite groups aside from the alternating and symmetric groups are the Mathieu groups, with the largest action on a set of size 24 (Dixon and Mortimer, 1996). Hence, for $n>24$, every 4- or 5-transitive voting rule must have either $S_{n}$ or $A_{n}$ as an automorphism group. Furthermore, the only 6-equitable groups are $S_{n}$ or $A_{n}$ (again, see Dixon and Mortimer, 1996). Hence, the result follows immediately from Lemma 4. 


\section{2-Equitable and 3-Equitable Rules}

In this section we show that for arbitrarily large $n$ there exist 3-equitable rules with coalitions of size $O(\sqrt{n} \log n)$, and 2-equitable rules with coalitions of size $\sqrt{n}$ (rounded up to the nearest integer), which, remarkably, sharply matches our lower bound of $\sqrt{n}$.

Theorem 6. Let the set of voters be of size $n=q^{2}+q+1$, for prime $q$. Then there is a 2 -equitable voting rule with a winning coalition of size exactly equal to $\sqrt{n}$, rounded up to the nearest integer.

More generally, a similar statement holds when $n=q^{2}+q+1$ and $q=p^{k}$ for some $k \geq 1$ and $p$ prime. The example in Figure 5 corresponds to the case $q=2$.

Proof. Let $\mathbb{F}_{q}$ denote the finite field with $q$ elements. ${ }^{16}$

Given a positive integer $m, \mathbb{F}_{q}^{m}$ is a vector space, where the scalars take values in $\mathbb{F}_{q}$ : it satisfies all the axioms that (say) $\mathbb{R}^{3}$ satisfies, but for scalars that are in $\mathbb{F}_{q}$ instead of $\mathbb{R}$. Indeed, much of the standard theory of linear algebra of $\mathbb{R}^{m}$ applies in this finite setting, and we will make use of it here.

In particular, we will make use of $G L(m, q)$, the group of invertible, $m \times m$ matrices with entries in $\mathbb{F}_{q}$. Here, again, the product of two matrices is calculated as usual, but addition and multiplication are taken modulo $q$. Since $\mathbb{F}_{q}^{m}$ is finite, each matrix in $G L(m, q)$ corresponds to a permutation of $\mathbb{F}_{q}^{m}$. As in the case of matrix multiplication on $\mathbb{R}^{m}$, these permutations preserve the 1-dimensional and 2-dimensional subspaces. Moreover, this group acts 2-transitively on the 1-dimensional subspaces, as any two non-colinear vectors $(u, v)$ can be completed to a basis of $\mathbb{F}_{q}^{m}$, and likewise starting from $\left(u^{\prime}, v^{\prime}\right)$; then any basis can be carried by an invertible matrix to any other basis.

With this established, we are ready to identify our set of voters with the set of 1-dimensional subspaces of $\mathbb{F}_{q}^{3}$. For each 2-dimensional subspace $U$ of $\mathbb{F}_{q}^{3}$, define the set $S_{U}$ of 1-dimensional

\footnotetext{
${ }^{16} \mathbb{F}_{q}$ is the set $\{0,1, \ldots, q-1\}$, equipped with the operations of addition and multiplication modulo $q$. The primality of $q$ is required to make multiplication invertible.
} 
subspaces (i.e., voters) contained in $U$. Let $\mathcal{W}$ be the collection of all such sets $S_{U}$, and define, using Lemma 1, a voting rule $f$ in which the sets $S_{U}$ are winning coalitions. We need to verify that any two winning coalitions $S_{U}$ and $S_{U^{\prime}}$ are non-disjoint. This simply follows from the fact that every pair of 2-dimensional subspaces intersects in some 1-dimensional subspace, and so it follows that each pair of such winning coalitions will have exactly one voter in common. ${ }^{17}$

A simple calculation shows that the winning coalitions are of size $q+1$. Since $\sqrt{n} \leq$ $q+1 \leq \sqrt{n}+1$, the claim follows.

To construct 3-equitable rules we will need the following lemma. It allows us to show, using the probabilistic method, that for small automorphism groups we can construct voting rules with small winning coalitions. This is useful for proving that there exist 3-equitable voting rules with small winning coalitions.

Lemma 5. Let $G$ be a group of $m$ permutations of $\{1, \ldots, n\}$. Then there is a neutral and positively responsive voting rule $f$ such that $G$ is a subgroup of $\mathrm{Aut}_{f}$, and $f$ has winning coalitions of size at most $2 \sqrt{n} \log m+2$.

We use this lemma to prove our theorem illustrating the existence of 3-equitable rules with small winning coalitions for arbitrarily large voter populations. We then return to prove the lemma.

Theorem 7. For $n$ such that $n-1$ is a prime power, there is a 3-equitable voting rule with a winning coalition of size at most $6 \sqrt{n} \log n$.

Proof. For $n$ such that $n-1$ is the power of some prime there is a 3-transitive group of permutations of $\{1, \ldots, n\}$ that is of size $m<n^{3}$. ${ }^{18}$ Hence, by Lemma 5 , there is a 3-equitable

\footnotetext{
${ }^{17}$ This is the reason that the winning coalitions of this rule are so small and proving a tight match to the lower bound.

${ }^{18}$ The group $P G L(2, n-1)$ acts 3 -transitively on the projective line over the field $\mathbb{F}_{n-1}$, and is of size $n(n-1)(n-2)<n^{3}$.
} 
voting rule for $n$ (i.e., a rule with a 3-transitive automorphism group) with a winning coalition of size at most $2 \sqrt{n} \log \left(n^{3}\right)=6 \sqrt{n} \log n$.

It is natural to conjecture that this probabilistic construction is not optimal, and that there exist 3-equitable rules with winning coalitions of size $O(\sqrt{n})$.

The heart of Lemma 5 is the following group-theoretic claim, which states that when $G$ is small then we can find a small set $S$ such that $g S$ and $S$ are non-disjoint for every $g \in G$. These sets $g S$ will be the winning coalitions used to prove Lemma 5 . The proof of this proposition uses the probabilistic method: we choose $S$ at random from some distribution, and show that with positive probability it has the desired property. This proves that there exists a deterministic $S$ with the desired property.

Proposition 1. Let a group $G$ of $m>2$ permutations of $\{1, \ldots, n\}$. Then there exists a set $S \subseteq\{1, \ldots, n\}$ with $|S| \leq 2 \sqrt{n} \log m+2$ such that $\forall g \in G$ we have $g S \cap S \neq \emptyset$.

Proof. To prove this, we will choose $S$ at random, and prove that it has the desired properties with positive probability. Let $\ell=\lceil\sqrt{n} \log |G|\rceil$. Let $S=S_{1} \cup S_{2}$, where $S_{1}$ is any subset of $X$ of size $\ell$, and $S_{2}$ is the union of $\ell$ elements of $X$, chosen independently from the uniform distribution. Hence $S$ includes at most $2 \ell \leq 2 \sqrt{n} \log |G|+2$ elements.

We now show that $\mathbb{P}(\forall g \in G: g S \cap S \neq \emptyset)>0$, and hence there is some set $S$ with the desired property. Note that for any particular $g \in G$, the distribution of $g S_{2}$ is identical to the distribution of $S_{2}$. Hence

$$
\begin{aligned}
\mathbb{P}(g S \cap S=\emptyset) & \leq \mathbb{P}\left(g S_{2} \cap S_{1}=\emptyset\right) \\
& =\mathbb{P}\left(S_{2} \cap S_{1}=\emptyset\right) \\
& =\left(\frac{n-\ell}{n}\right)^{\ell} \\
& \leq e^{-\ell^{2} / n} \\
& \leq e^{-(\log m)^{2}}
\end{aligned}
$$


Thus, the probability that there is some $g \in G$ for which $g S \cap S=\emptyset$ is, by taking a union bound, at most

$$
m e^{-(\log |G|)^{2}}
$$

which is strictly less than 1 for $m>2$.

We are finally ready to prove Lemma 5.

Proof of Lemma 5. Let $S$ be the subset of $\{1, \ldots, n\}$ given by Proposition 1 . Let $\mathcal{W}$ be the collection of sets of the form $g S$, where $g \in G$. This is a collection of pairwise non-disjoint sets, since if $g S$ and $h S$ intersect then so do $h^{-1} g S$ and $S$, which is impossible by the defining property of $S$. Since $|S|=2 \sqrt{n} \log m+2$ the claim follows from Lemma 1. 


\section{References}

P. J. Cameron, P. M. Neumann, and D. N. Teague. On the degrees of primitive permutation groups. Mathematische Zeitschrift, 180(3):141-149, 1982. ISSN 1432-1823.

E. Cantillon and A. Rangel. A graphical analysis of some basic results in social choice. Social Choice and Welfare, 19(3):587-611, 2002.

P. Dasgupta and E. Maskin. The existence of equilibrium in discontinuous economic games, i: Theory. Review of Economic Studies, 53(1):1-26, 1986.

J. D. Dixon and B. Mortimer. Permutation Groups, volume 163. Springer Science \& Business Media, 1996.

P. Dubey and L. S. Shapley. Mathematical properties of the banzhaf power index. Mathematics of Operations Research, 4(2):99-131, 1979.

M. Fey. May's theorem with an infinite population. Social Choice and Welfare, 23(2):275-293, 2004.

R. Goodin and C. List. A conditional defense of plurality rule: Generalizing may's theorem in a restricted informational environment. American Journal of Political Science, 50(4): 940-949, 2006.

K. O. May. A set of independent necessary and sufficient conditions for simple majority decision. Econometrica, 20(4):680-684, 1952.

A. J. McGann, C. A. Smith, M. Latner, and A. Keena. Gerrymandering in America: The House of Representatives, the Supreme Court, and the Future of Popular Sovereignty. Cambridge University Press, 2016.

E. Mossel and R. O'Donnell. Recursive reconstruction on periodic trees. Random Structures E Algorithms, 13(1):81-97, 1998. 
J. Nash. Non-cooperative games. Annals of Mathematics, pages 286-295, 1951.

E. W. Packel. Transitive permutation groups and equipotent voting rules. Mathematical Social Sciences, 1(1):93-100, 1980.

H.-O. Peitgen, H. Jürgens, and D. Saupe. Chaos and Fractals: New Frontiers of Science. Springer, 1993.

L. Penrose. The elementary statistics of majority voting. Journal of the Royal Statistical Society, 109:53-57, 1946.

W. Reiker. The Theory of Political Coalitions. New Haven and London: Yale University Press, 1962.

J. J. Rotman. An Introduction to the Theory of Groups, volume 148. Springer Science \& Business Media, 2012.

H. R. Varian. Equity, envy, and efficiency. Journal of Economic Theory, 9:63-91, 1974.

K. Życzkowski and W. Słomczyński. Square root voting system, optimal threshold and $\pi$. In Voting Power and Procedures, pages 127-146. Springer, 2014. 